\title{
GENERALIZED EMDEN-FOWLER EQUATIONS OF SUBCRITICAL GROWTH
}

\author{
WOLFGANG ROTHER
}

(Received 1 November 1990)

Communicated by E. N. Dancer

\begin{abstract}
The existence of positive solutions, vanishing at infinity, for the semilinear eigenvalue problem $\mathrm{L} u=\lambda \cdot f(x, y)$ in $\mathbb{R}^{N}$ is obtained, where $L$ is a strictly elliptic operator. The function $f$ is assumed to be of subcritical growth with respect to the variable $u$.
\end{abstract}

1991 Mathematics subject classification (Amer. Math. Soc.): 35 J 60.

\section{Introduction}

In a recent paper, Noussair and Swanson [3] study the semilinear problem

$$
\mathrm{L} u=\lambda \cdot f(x, u) \text { in } \mathbb{R}^{N}(N \geq 3),
$$

where $\mathrm{L} u=-\sum_{l, m=1}^{N} \partial_{m}\left(a_{l m}(x) \cdot \partial_{l} u\right)$ is a strictly elliptic operator, meaning that there exists a positive constant $a_{0}$ such that

$$
\sum_{l, m=1}^{N} a_{l m}(x) \xi_{l} \xi_{m} \geq a_{0}|\xi|^{2}
$$

holds for all $\xi \in \mathbb{R}^{N}$ and almost all $x \in \mathbb{R}^{N}$.

The function $f$ is supposed to satisfy

$$
0 \leq f(x, t) \leq \sum_{i=1}^{I} f_{i}(x) \cdot t^{\gamma i}
$$

(C) 1993 Australian Mathematical Society $0263-6115 / 93 \$ A 2.00+0.00$ 
for all $x \in \mathbb{R}^{N}$ and $t \geq 0$, where the constants $\gamma_{i}$

$$
(i=1, \ldots, I) \text { satisfy } 1<\gamma_{i}<\frac{N+2}{N-2} .
$$

Furthermore, the authors of [3] assume

(A1) that the function $f$ is locally Hölder continuous on $\mathbb{R}^{N} \times[0, \infty[$, and that the functions $a_{l m}$ satisfy $a_{l m} \in C_{l o c}^{1+\alpha}\left(\mathbb{R}^{N}\right)$ and $\left\|a_{l m}\right\|_{c^{0, \alpha}\left(\overline{\mathbf{R}^{N}}\right)}<$ $\infty$ for a constant $0<\alpha<1$;

(A2) that there exists an open subset $\Omega \neq \varnothing$ of $\mathbb{R}^{N}$ such that $f(x, t)>0$ holds on $\Omega \times] 0, \infty[$

(A3) that the functions $f_{i}(i=1, \ldots, I)$ are nonnegative, bounded and continuous such that $f_{i} \in L^{q_{i}}\left(\mathbb{R}^{N}\right)$ holds for a constant $1<q_{i}<$ $\frac{2 N}{2 N-(n-2) \cdot\left(1+\gamma_{i}\right)}$

(A4) and that $t \cdot f(x, t) \geq C \cdot F(x, t)$ holds for all $x \in \mathbb{R}^{N}$ and $t \geq 0$, where $C$ is a positive constant and $F(x, t)=\int_{0}^{t} f(x, s) d s$.

Then, it is shown (see [3, Theorem 1]) that there is a positive solution pair $(\lambda, u)$ of equation (1) such that $u \in L^{Q}\left(\mathbb{R}^{N}\right)$ holds for all $2 N /(N-2) \leq$ $Q \leq \infty$, and $|\nabla u(x)|$ as well $u(x)$ has uniform limit zero as $|x| \rightarrow \infty$. It should be mentioned that the authors of [3] do not require explicitly that the functions $a_{l m}$ are uniformly Hölder continuous on $\mathbb{R}^{N}$. But, when they show that $|\nabla u(x)|$ vanishes uniformly at infinity (see $[3$, p. 58]), they use the estimate (8.86) in [2] which depends on $\max \left\|a_{l m}\right\|_{C^{0, a}}$; so the proof only works if $\left\|a_{l m}\right\|_{C^{0, \alpha}\left(\overline{\mathbf{R}^{n}}\right)}<\infty$.

The aim of this paper is to show that assumption (A4) is superfluous and that the assumptions (A2) and (A3) can be weakened considerably (see Corollary 1). Furthermore, we will prove the existence of a $\lambda>0$ and a weak solution $u>0$ of equation (1) if (A1) is replaced by the following condition:

(B1) The function $f: \mathbb{R}^{N} \times\left[0, \infty\left[\mapsto \mathbb{R}_{+}\right.\right.$is measurable and, for $x \in \mathbb{R}^{N}$,

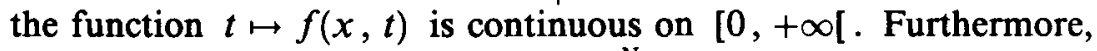
the functions $a_{l m}$ satisfy $a_{l m} \in L^{\infty}\left(\mathbb{R}^{N}\right)$.

Instead of (A2), we will assume:

(B2) There exist an open subset $\Omega \neq \varnothing$ of $\mathbb{R}^{N}$ and constants $0 \leq \delta_{0}<$ $\delta_{1} \leq \infty$ such that $f(x, t)>0$ holds on $\left.\Omega \times\right] \delta_{0}, \delta_{1}[$.

Assumption (A3) will be replaced by

(B3) The functions $f_{i}$ are nonnegative and for each $i=1, \ldots, I$ there exists a constant $0<\varepsilon_{i}<\infty$ such that $f_{i} \in L^{q}\left(\mathbb{R}^{N}\right)$ holds for all $q \in\left[p_{i}, p_{i}+\varepsilon_{i}\right]$, where $p_{i}=\frac{2 N}{2 N-(N-2) \cdot\left(1+y_{i}\right)}$.

Then, we will prove the following 
THEOREM 1. Suppose the operator $L$ is strictly elliptic and the assumption (2), (3) and (B1)-(B3) are fulfilled. Then there exists a constant $\lambda>0$ and a positive function $u$ such that equation (1) holds in the weak sense. Moreover, the function $u$ is locally Holder continuous, satisfies $u \in L^{Q}\left(\mathbb{R}^{N}\right)$ for all $2 N /(N-2) \leq Q \leq \infty$, and vanishes uniformly at infinity.

COROLLARY 1. Suppose that the assumptions of Theorem 1 are satisfied, that (A1) is fulfilled and $\varepsilon_{i}>N-p_{i}$ holds for $i=1, \ldots, I$. Then, the function $u$ satisfies $u \in C^{2}\left(\mathbb{R}^{N}\right)$, and the pair $(\lambda, u)$ solves equation (1) in the classical sense. Moreover, $|\nabla u(x)| \rightarrow 0$ uniformly as $|x| \rightarrow \infty$.

Our method of proof is different from that used by Noussair and Swanson. The authors of [3] minimize the functional

$$
\mathrm{I}_{k}(u)=\frac{1}{2} \cdot \int_{\mathbf{R}^{N}}\left(\sum_{l, m=1}^{N} a_{l m} \cdot \partial_{l} u \cdot \partial_{m} u+\frac{1}{k} \cdot u^{2}\right) d x \quad(k \in \mathbb{N})
$$

subject to the constraints $u \in H^{1}\left(\mathbb{R}^{N}\right)$ and $\int_{\mathbb{R}^{N}} F(x, u(x)) d x=1$, and show that the infimum is attained by a function $u_{k} \in H^{1}\left(\mathbb{R}^{N}\right)$ satisfying

$$
\int\left(\sum_{l, m=1}^{N} a_{l m} \cdot \partial_{l} u_{k} \cdot \partial_{m} v+\frac{1}{k} \cdot u_{k} \cdot v\right) d x=\lambda_{k} \cdot \int f\left(x, u_{k}(x)\right) \cdot v(x) d x
$$

for all $v \in H^{1}\left(\mathbb{R}^{N}\right)$ and a constant $\lambda_{k}>0$.

Then, making essential use of (A4), the authors show that a subsequence of $\left(\lambda_{k}, u_{k}\right)$ converges to a solution $(\lambda, u)(\lambda>0, u>0)$ of equation (1).

In contrast to this method, we will maximize the functional

$$
J(u)=\int F(x, u(x)) d x /\left(\|u\|_{L}^{2}+\|u\|_{L}^{2^{*}}\right)
$$

on $\widehat{H}^{1}=\widehat{H}^{1}\left(\mathbb{R}^{N}\right)=\left\{u \in L^{2^{*}}\left(\mathbb{R}^{N}\right) \mid \nabla u \in\left(L^{2}\left(\mathbb{R}^{N}\right)\right)^{N}\right\}$, where

$$
2^{*}=2 N /(N-2) \text { and }\|u\|_{L}^{2}=\sum_{l, m=1}^{N} \int a_{l m} \cdot \partial_{l} u \cdot \partial_{m} u d x ;
$$

so, we need not assume (A4).

To prove that the function $u$ is in Theorem 1 satisfies $u \in L^{Q}$ for all $2^{*} \leq Q \leq \infty$, we will use a method of Stampacchia [4] and not the device of Brézis and Kato [1]; so we need not assume that the functions $f_{i}$ satisfy $f_{i} \in L^{q}\left(\mathbb{R}^{N}\right)$ for some $q<p_{i}$. 


\section{Preliminaries}

By $L^{p}=L^{p}\left(\mathbb{R}^{N}\right) \quad(1 \leq p \leq \infty)$, we denote the usual Lebesgue space and $\|\cdot\|_{p}$ is the norm on $L^{p}$. For $u, v \in \widehat{H}^{1}$, we define

$$
\langle u, v\rangle_{L}=\sum_{l, m=1}^{N} \int a_{l m}(x) \cdot \partial_{l} u(x) \cdot \partial_{m} v(x) d x
$$

and $\|u\|_{L}=\langle u, u\rangle_{L}^{1 / 2}$.

Since $L$ is strictly elliptic, we conclude from the Sobolev inequality that there is a constant $C_{0}>0$ such that

$$
\|u\|_{2^{*}} \leq C_{0} \cdot\|u\|_{L} \text { holds for all } u \in \widehat{H}^{1} .
$$

Hence, $\widehat{H}^{1}$ is a Hilbert space with scalar product $\langle\cdot, \cdot\rangle_{L}$. The function $u \in$ $\widehat{H}^{1}$ is called a weak solution of equation (1), if $\langle u, v\rangle_{L}=\lambda \cdot \int f(x, u(x))$. $v(x) d x$ holds for all $v \in \widehat{H}^{1}$.

For $x \in \mathbb{R}^{N}$ and $t<0$, we define $f(x, t)=0$. Moreover, we set $F(x, t)=\int_{0}^{t} f(x, s) d s$ for $t \in \mathbb{R}$ and $x \in \mathbb{R}^{N}$. Then, it follows that

$$
0 \leq F(x, t) \leq \sum_{i=1}^{I}\left(1+\gamma_{i}\right)^{-1} \cdot f_{i}(x) \cdot t_{+}^{\left(1+\gamma_{i}\right)}
$$

holds on $\mathbb{R}^{N} \times \mathbb{R}$, where $t_{+}=\max (t, 0)$.

From assumption (B2), we conclude that

$$
F(x, t)>0 \text { holds on } \Omega \times] \delta_{0}, \infty[\text {. }
$$

Further, from (4), (5) and Hölder's inequality, we obtain the following

LEMMA 1. For $i=1, \ldots, I$, the constant $p_{i}$ may be chosen as in (B3). Then, for $0 \leq R<\infty$ and $u \in \widehat{H}^{1}$, we have

$$
\int_{|x| \geq R} F(x, u(x)) d x \leq \sum_{i=1}^{I}\left(1+\gamma_{i}\right)^{-1} \cdot\left(\int_{|x| \geq R}\left|f_{i}\right|^{p_{i}} d x\right)^{1 / p_{i}} \cdot\left(C_{0} \cdot\|u\|_{L}\right)^{1+\gamma_{i}} \text {. }
$$

Lemma 1 shows that $J(u)$ is well defined for all $0 \neq u \in \hat{H}^{1}$. Furthermore, since $2<1+\gamma_{i}<2^{*}$, Lemma 1 implies the existence of a constant $S_{1}<\infty$ such that

$$
J(u) \leq S_{1} \text { holds for all } 0 \neq u \in \widehat{H}^{1} .
$$

Thus, $\mathscr{S}=\sup _{0 \neq u \in \widehat{H}^{1}} J(u)$ is a well defined real number. 
The function $\varphi_{0} \in C_{0}^{\infty}$ may be chosen such that $\operatorname{supp} \varphi_{0} \subset \Omega$ and $\sup _{x} \varphi_{0}(x)>\delta_{0}$, where $\delta_{0}$ is the constant from (B2). Then, according to (6), we see that

$$
S_{0}=\frac{1}{2} \cdot J\left(\varphi_{0}\right)>0 \text { and } \mathscr{S}>S_{0}
$$

\section{Proof of the results}

In the following, we always suppose that the assumptions of Theorem 1 are fulfilled.

Proof OF TheOREM 1. Let $\left(u_{n}\right)_{n} \subset \widehat{H}^{1}$ be a sequence such that $u_{n} \neq 0$ and $J\left(u_{n}\right) \rightarrow \mathscr{S}$ as $n \rightarrow \infty$.

According to $(8)$, we may assume without restriction that

$$
J\left(u_{n}\right) \geq S_{0} \text { holds for all } n .
$$

Since $J\left(\left(u_{n}\right)_{+}\right) \geq J\left(u_{n}\right)$, we may assume further that $u_{n} \geq 0$. Then, Lemma 1 implies

$$
S_{0} \cdot\left(\left\|u_{n}\right\|_{L}^{2}+\left\|u_{n}\right\|_{L}^{2^{*}}\right) \leq C \cdot \sum_{i=1}^{I}\left\|u_{n}\right\|_{L}^{1+\gamma_{i}}
$$

for all $n$ and a constant $C$.

Hence, there exist constants $0<U_{0}<U_{1}<\infty$ such that

$$
U_{0} \leq\left\|u_{n}\right\|_{L} \leq U_{1} \text { holds for all } n \text {. }
$$

So, we can find a subsequence of $\left(u_{n}\right)_{n}$, still denoted by $\left(u_{n}\right)_{n}$, and $u$, $0 \leq u \in \widehat{H}^{1}$, such that $u_{n} \underset{w}{u}$ in $\widehat{H}^{1}$. According to Lemma 1 and (10), for each $\varepsilon>0$, there exists a constant $R_{\varepsilon}>0$ such that $\int_{|x| \geq R_{\varepsilon}} F(x, u(x)) d x \leq$ $\varepsilon$, and

$$
\int_{|x| \geq R_{\varepsilon}} F\left(x, u_{n}(x)\right) d x \leq \varepsilon
$$

holds for all $n$.

Using the Rellich-Kondrachov theorem and the fact that $f_{i} \in L^{p_{i}+\varepsilon_{i}}$, it can be shown that

$$
\int_{|x|<R_{\varepsilon}} F\left(x, u_{n}(x)\right) d x \rightarrow \int_{|x|<R_{\varepsilon}} F(x, u(x)) d x
$$

as $n \rightarrow \infty$. But (11) and (12) then imply

$$
\int F\left(x, u_{n}(x)\right) d x \rightarrow \int F(x, u(x)) d x .
$$


From (9), (10) and (13) it follows that

$$
\int F(x, u(x)) d x \geq S_{0} \cdot\left(U_{0}^{2}+U_{0}^{2^{*}}\right)>0
$$

and therefore, that $u \neq 0$.

From the uniform boundedness principle, we obtain

$$
\|u\|_{L}^{2}+\|u\|_{L}^{2^{*}} \leq \liminf \left(\left\|u_{n}\right\|_{L}^{2}+\left\|u_{n}\right\|_{L}^{2^{*}}\right) .
$$

Now, (13) and (14) imply $J(u) \geq \lim \sup J\left(u_{n}\right)=\mathscr{S}$ and, consequently, that $J(u)=\mathscr{S}$.

For any $v \in \hat{H}^{1}$, we can find an $\varepsilon_{0}=\varepsilon_{0}(v)$, such that $\|u+\varepsilon \cdot v\|_{L}>0$ holds for all $|\varepsilon|<\varepsilon_{0}$. For $\left.\varepsilon \in\right]-\varepsilon_{0}, \varepsilon_{0}[$, we define $\eta(\varepsilon)=J(u+\varepsilon \cdot v)$. But $\eta^{\prime}(0)=0$ then implies

$$
\begin{gathered}
\langle u, v\rangle_{L}=\lambda \cdot \int f(x, u(x)) \cdot v(x) d x, \text { where } \\
\lambda=\left(\|u\|_{L}^{2}+\|u\|_{L}^{2^{*}}\right) \cdot\left(\int F(x, u(x)) d x \cdot\left(2+2^{*} \cdot\|u\|_{L}^{2^{*}-2}\right)\right)^{-1}>0 .
\end{gathered}
$$

For each $i=1, \ldots, I$, there exists a constant $\varepsilon_{i}^{*}$, satisfying $0<\varepsilon_{i}^{*}<$ $\frac{2 N}{1+\gamma_{i}}-(N-2)$, such that

$$
p_{i}+\varepsilon_{i}=2 N /\left(2 N-\left(N-2+\varepsilon_{i}^{*}\right) \cdot\left(1+\gamma_{i}\right)\right) .
$$

For $k>0$, let $u_{k}=(u-k)_{+}$and $A(k)=\{x \mid u(x)>k\}$. Then, it is well known that $u_{k} \in \widehat{H}^{1}$, that $\partial_{l} u_{k}=\partial_{l} u$ holds on $A(k)$ and that $\partial_{l} u_{k}=0$ on $\mathbb{R}^{N} \backslash A(k)$. Inserting $v=u_{k}$ in (15), we conclude from (B3) and (4):

$$
\begin{aligned}
& \left(\int_{A(k)}(u-k)^{2^{*}} d x\right)^{2 / 2^{*}} \leq C_{0}^{2} \cdot \sum_{i=1}^{I}\left\|f_{i}\right\|_{p_{i}+\varepsilon_{i}} \\
& \cdot\left(\int_{A(k)}|u(x)|^{2 N /\left((N-2)+\varepsilon_{i}^{*}\right)} d x\right)^{\left((N-2)+\varepsilon_{i}^{*}\right) \cdot\left(1+\gamma_{i}\right) / 2 N} \\
& \leq C_{0}^{2} \cdot \sum_{i=1}^{I}\left\|f_{i}\right\|_{p_{i}+\varepsilon_{i}} \cdot\|u\|_{2^{*}}^{1+\gamma_{i}} \cdot(\operatorname{meas} A(k))^{\varepsilon_{i}^{*} \cdot\left(1+\gamma_{i}\right) / 2 N} .
\end{aligned}
$$

Furthermore, for $h>k>0$, we obtain

$$
\left(\int_{A(k)}(u-k)^{2^{*}} d x\right)^{2 / 2^{*}} \geq(h-k)^{2} \cdot(\text { meas } A(h))^{2 / 2^{*}} .
$$


In the following, the constant $k_{0}>0$ may be chosen such that meas $A\left(k_{0}\right)$ $\leq 1$; and the constant $\gamma_{0}$ may be defined by $\gamma_{0}=\min \left\{\gamma_{1}, \ldots, \gamma_{I}\right\}$. Furthermore, $\varepsilon_{0}^{*}$ may satisfy $0<\varepsilon_{0}^{*} \leq \min \left\{\varepsilon_{1}^{*}, \ldots, \varepsilon_{I}^{*}\right\}, \varepsilon_{0}^{*}<2 \cdot(N-2) /\left(1+\gamma_{0}\right)$ and

$$
n \cdot \varepsilon_{0}^{*} \neq 2 \cdot(N-2) /\left(1+\gamma_{0}\right) \text { for all } n \in \mathbb{N} \text {. }
$$

Then, there exists a constant $n_{0} \in \mathbb{N}$ such that

$$
n_{0} \cdot \varepsilon_{0}^{*}<2 \cdot(N-2) /\left(1+\gamma_{0}\right) \text { and }\left(n_{0}+1\right) \cdot \varepsilon_{0}^{*}>2 \cdot(N-2) /\left(1+\gamma_{0}\right) \text {. }
$$

Hence, for $h>k \geq k_{0}$, we conclude from (16) and (17):

$$
\text { meas } A(h) \leq C \cdot(h-k)^{-2^{*}} \cdot(\text { meas } A(k))^{\left(1+\gamma_{0}\right) \cdot \varepsilon_{0}^{*} / 2 \cdot(N-2)},
$$

where the constant $C$ is independent of $h$ and $k$. By part (iii) of $[4$, Lemma 4.1], it follows that

$$
\text { meas } A(h) \leq K \cdot h^{-\mu}
$$

holds for a constant $K>0$ and all $h \geq k_{0}$, where

$$
\mu=4 N /\left(2 \cdot(N-2)-\left(1+\gamma_{0}\right) \cdot \varepsilon_{0}^{*}\right)
$$

For $p \geq 2^{*}$, we have

$$
\begin{aligned}
\int(u(x))^{p} d x= & \int_{\left\{u \leq k_{0}\right\}}(u(x))^{p} d x+\int_{0}^{\infty} p \cdot \sigma^{p-1} \cdot \operatorname{meas}\left(\left\{u>k_{0}\right\} \cap\{u>\sigma\}\right) d \sigma \\
\leq & k_{0}^{p-2^{*}} \cdot\|u\|_{2^{*}}^{2^{*}}+k_{0}^{p} \cdot \operatorname{meas}\left\{u>k_{0}\right\} \\
& +\int_{k_{0}}^{\infty} p \cdot \sigma^{p-1} \text { meas }\{u>\sigma\} d \sigma
\end{aligned}
$$

(see [2, Lemma 9.7]).

Thus, (18) shows that $u \in L^{p}$ for all $2^{*} \leq p<\mu$. In particular, we see that $u \in L^{p_{1}}$ for

$$
p_{1}=4 N /\left(2 \cdot(N-2)-2 \cdot \varepsilon_{0}^{*}\right)=2 N /\left((N-2)-\varepsilon_{0}^{*}\right) .
$$

Moreover, by induction, it can be shown that

$$
u \in L^{p_{n}} \text { holds for all } n=1, \ldots, n_{0},
$$

where $p_{n}=2 N /\left((N-2)-n \cdot \varepsilon_{0}^{*}\right)$.

Using the fact that $u \in L^{p_{n_{0}}}$ and proceeding as above, we obtain

$$
\text { meas } A(h) \leq C \cdot(h-k)^{-2^{*}} \cdot(\text { meas } A(k))^{\left(n_{0}+1\right) \cdot\left(1+\gamma_{0}\right) \cdot \varepsilon_{0}^{*} / 2 \cdot(N-2)}
$$

for all $h>k \geq k_{0}$. But now, part (i) of [4, Lemma 4.1] implies $u \in L^{\infty}$; so, we have $u \in L^{Q}$ for all $2^{*} \leq Q \leq \infty$. 
The function $h: \mathbb{R}^{N} \times \mathbb{R} \rightarrow[0, \infty[$ may be defined by $h(x, t)=f(x, t) / t$ if $x \in \mathbb{R}^{N}$ and $t>0$, and by $h(x, t)=0$ otherwise. Then, by (2), we obtain that

$$
0 \leq h(x, t) \leq \sum_{i=1}^{I} f_{i}(x) \cdot t_{+}^{\gamma_{i}-1}
$$

holds for all $x \in \mathbb{R}^{N}$ and $t \in \mathbb{R}$.

The constant $\alpha_{0}$ may be chosen such that $1<\alpha_{0}<\gamma_{0}$, and $p_{0}$ may be defined by $p_{0}=2 N /\left(2 N-(N-2) \cdot\left(1+\alpha_{0}\right)\right)$. Further, by $r_{i}(i=1, \ldots, I)$, we denote the constant

$$
r_{i}=2^{*} \cdot\left(\gamma_{i}-1\right) /\left(\gamma_{i}-\alpha_{0}\right) .
$$

Since $u \in L^{r_{i}}$, we obtain by Hölder's inequality:

$$
\left\|f_{i} \cdot u^{\gamma_{i}-1}\right\|_{p_{0}} \leq\left\|f_{i}\right\|_{p_{i}} \cdot\|u\|_{r_{i}}^{\gamma_{i}-1} \text { for all } i=1, \ldots, I \text {. }
$$

Hence, (19) implies $h(\cdot, u(\cdot)) \in L^{p_{0}}$.

From (15), it follows that

$$
\langle u, v\rangle_{L}-\lambda \cdot \int h(x, u(x)) \cdot u(x) \cdot v(x) d x=0
$$

holds for all $v \in \widehat{H}^{1}$.

Then, because $p_{0}>N / 2$, we conclude from [4, Corollary 8.1] that $u$ is positive. Since $u$ is bounded and $f(x, u(x))=h(x, u(x)) \cdot u(x)$, we see that $f(\cdot, u(\cdot)) \in L^{p_{0}}$. Then, using [2, Theorem 8.24] and proceeding as in $[3$, p. 58], it can be shown that $u$ is locally Hölder continuous and $u(x) \rightarrow 0$ uniformly as $|x| \rightarrow \infty$.

Proof of Corollary 1. Now, we assume additionally that (A1) is satisfied and $p_{i}+\varepsilon_{i}>N$ holds for $i=1, \ldots, I$. The function $u$ and the constant $\lambda$ may be chosen as in Theorem 1 . Then, the function $g(x)=$ $\lambda \cdot f(x, u(x))$ is locally Hölder continuous on $\mathbb{R}^{N}$. In particular, we see that $g \in L_{\mathrm{loc}}^{2}$. Hence, [2, Theorem 8.8] implies $u \in W_{\mathrm{loc}}^{2,2}$ and

$$
-\sum_{l, m=1}^{N}\left(a_{l m} \cdot \partial_{m l} u+\partial_{m} a_{l m} \cdot \partial_{l} u\right)=g \text { a.e. in } \mathbb{R}^{N} \text {. }
$$

Now, we apply [2, Theorem 9.19] and conclude that $u \in C_{\text {loc }}^{2}$. Furthermore, we see that $(\lambda, u)$ solves equation (1) in the classical sense.

The constant $q_{0}$ may be chosen such that

$$
N<q_{0} \leq \min \left\{p_{1}+\varepsilon_{1}, \ldots \ldots, p_{I}+\varepsilon_{I}\right\},
$$


and we let $B_{\rho}(x)$ denote the ball in $\mathbb{R}^{N}$ with centre $x$ and radius $\rho$. Then, using [2, Problem 4.8(b)] and the remark after $(4.46)$ in $[2$, p. 70], and proceeding as in the proof of [2, Theorem 8.32$]$, it can be shown there exists a constant $C$, independent of $x$, such that

$$
\|\left. u\right|_{C^{1, \nu}\left(B_{1}(x)\right)} \leq C \cdot\left(\|u\|_{C^{0}\left(B_{2}(x)\right)}+\|g\|_{L^{q_{0}}\left(B_{2}(x)\right)}\right),
$$

where $0<\nu<\min \left\{\alpha,\left(q_{0}-N\right) / q_{0}\right\}$. Since $u$ vanishes uniformly at infinity and

$$
\|g\|_{L^{q_{0}}\left(B_{2}(x)\right)} \leq K \cdot \sum_{i=1}^{I}\left\|f_{i}\right\|_{L^{p_{i}+\varepsilon_{i}}\left(B_{2}(x)\right)}
$$

holds for a constant $K$ which is independent of $x$, we conclude from (20) that $|\nabla u(x)|$ has uniform limit zero as $|x| \rightarrow \infty$.

\section{References}

[1] H. Brézis and T. Kato, 'Remarks on the Schrödinger operator with singular complex potentials', J. Math. Pures Appl. 58 (1979), 137-151.

[2] D. Gilbarg and N. S. Trudinger, Elliptic partial differential equations of second order (Springer, Berlin, 1983).

[3] E. S. Noussair and C. A. Swanson, 'An $L^{q}\left(\mathbf{R}^{N}\right)$-theory of subcritical semilinear elliptic problems', J. Differential Equations 84 (1990), 52-61.

[4] G. Stampacchia, 'Le probléme de Dirichlet pour les équations elliptiques du second ordre à coefficients discontinus', Ann. Inst. Fourier, (Grenoble) 15 (1965), 189-258.

University of Bayreuth

P. O. B. 101251

W-8580 Bayreuth

Federal Republic of Germany 